\title{
First spectroscopy of a short-hard GRB: the environment of a compact object merger
}

\author{
Antonio de Ugarte Postigo ${ }^{1,2}$, Christina C. Thöne ${ }^{2}$, \\ Antonia Rowllinson ${ }^{3}$, Rubén García Benito ${ }^{1}$, Andrew J. Levan ${ }^{4}$, \\ Javier Gorosabel ${ }^{1,5}$, Paolo Goldoni ${ }^{6}$ and Steve Schulze ${ }^{7,8}$ \\ ${ }^{1}$ IAA-CSIC, Glorieta de la Astronomía, s/n, Granada, Spain, email: deugarte@iaa.es \\ ${ }^{2}$ DARK/NBI, U. of Copenhagen, Juliane Maries Vej 30, Copenhagen $\varnothing$, Denmark \\ ${ }^{3}$ Ast. Inst. 'Anton Pannekoek', U. Amsterdam, 1090 GE Amsterdam, The Netherlands \\ ${ }^{4}$ Dept. of Physics, U. Warwick, Coventry, UK \\ ${ }^{5}$ Unidad Asociada Grupo de Ciencias Planetarias, UPV/EHU - IAA-CSIC, Alameda de \\ Urquijo 36-5, Bilbao, Spain \\ ${ }^{6}$ APC, U. Paris Diderot, CNRS/IN2P3, CEA/IRFU, Obs. Paris, Sorbonne Paris Cité, France \\ ${ }^{7}$ Pontificia Universidad Católida de Chile, Dep. de Astronomía y Astrofísica, Santiago 22, Chile \\ ${ }^{8}$ Millenium Center for Supernova Science, Chile
}

\begin{abstract}
Short gamma-ray bursts (GRBs) are an extremely elusive family of cosmic explosions. They are thought to be related to the violent merger of compact objects (such as a neutron stars or black holes). Their optical counterparts were not discovered until 2005, and since then, there had been no successful spectroscopic observations. Here we present the first spectra of a short GRB, which we use to study the environment and derive implications on the progenitors of these cosmic explosions. This poster is based on the work by de Ugarte Postigo et al. (2014).
\end{abstract}

\section{Discovery and observations of GRB 130603B}

GRB 130603B was detected by the Swift mission (Melandri et al. 2013). It had a duration of $0.18 \pm 0.02 \mathrm{~s}$, a hardness ratio of $\mathrm{F}(50-100 \mathrm{keV}) / \mathrm{F}(25-50 \mathrm{keV})=2.25 \pm 0.16$, a peak energy of $895 \pm 135 \mathrm{keV}$, negligible spectral lags $(-2.5 \pm 0.7 \mathrm{~ms}$ between the $25-50$ $\mathrm{keV}$ and the 100-350 keV bands) and no extended emission, unambiguously classifying as a prototypical short-hard GRB.

Following the discovery by Swift we started a follow-up campaign and discovered an optical counterpart with the $4.2 \mathrm{~m}$ WHT (Levan et al. 2014) and the $2.5 \mathrm{~m}$ NOT (de Ugarte Postigo et al. 2013). Optical/NIR follow-up was performed with $1.23 \mathrm{~m}$ CAHA, GTC, NOT, WHT TNG VLT and Gemini telescopes. X-ray data was obtained with XRT onboard Swift. Late observations with $H S T$ revealed a tidally disrupted late-type host galaxy. A fit to the spectral energy distribution shows a line of sight extinction of $\mathrm{A}_{V}=0.86 \pm 0.15 \mathrm{mag}$.

Observations from $H S T$ obtained 9 days after the GRB revealed an excess in the nIR emission consistent with an r-process kilonova, which had been predicted to happen during the merger of two compact objects. This is the first time that such an emission has been seen and would confirm the merger model (Tanvir et al. 2013; Berger et al. 2013). 


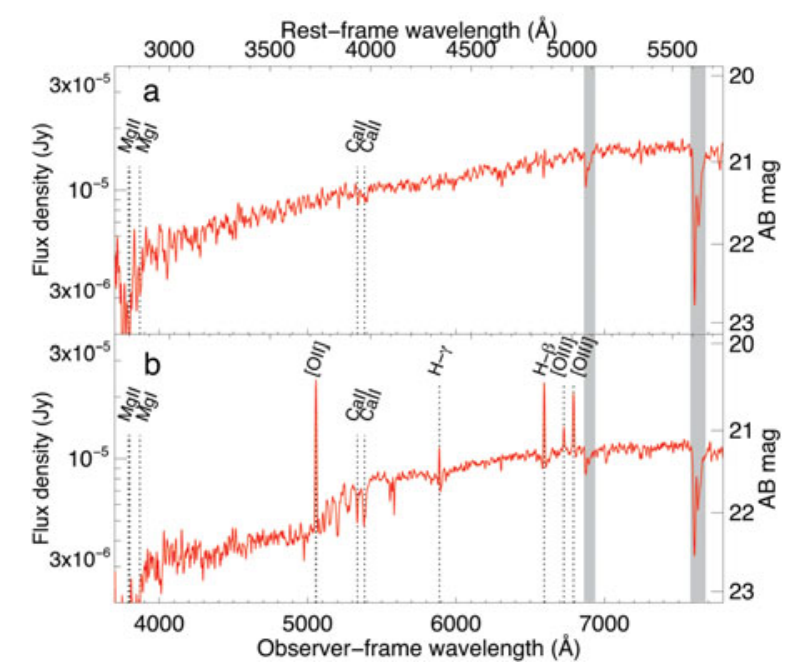

Figure 1. OSIRIS/GTC spectrum of the afterglow of GRB 130603B (top) and its host galaxy (bottom), showing both absorption and emission features. From de Ugarte Postigo et al. (2014).

\section{Spectroscopy}

The first spectrum of this short GRB was obtained with OSIRIS, at the Gran Telescopio Canarias (GTC), 7 hrs after the GRB onset. It revealed both emission and absorption features at a common redshift of $0.3565 \pm 0.0002$ (Thöne et al. 2013). We obtained 3 more spectra in the hours that followed from X-shooter and FORS2 at the VLT, and ACAM at the WHT.

The higher spectral resolution of the X-shooter spectra allows us to study the dynamics of the host. The spectra show absorption features of $\mathrm{MgII}, \mathrm{MgI}, \mathrm{CaII}$, and $\mathrm{NaI}$ in the line of sight of the GRB. Line widths range from 100-180 $\mathrm{km} \mathrm{s}^{-1}$. The strength of these lines is slightly larger than typical long GRBs, indicating a dense line-of-sight environment. Emission features present two components. The main one is broad $\left(92 \mathrm{~km} \mathrm{~s}^{-1}\right)$ and matches the redshift of the absorption features. The weaker system is blue-shifted by 120 $\mathrm{km} \mathrm{s}^{-1}$.

\section{Host galaxy}

Using the different spectra that were obtained, and performing different extractions in several regions, we performed a resolved study of the host. The galaxy is dominated by an 1 Gyr old population, but is still forming stars at a rate of 4.8 solar masses per year. The metallicity is 0.5 solar both at the location of the GRB (in a spiral arm) and the core, being slightly higher at the opposite end. The characteristics of the host and the location of the GRB within it favour a compact binary merger progenitor close to its birth place either due to a short merger time or low natal kick.

\section{References}

Berger, E., et al. 2013, ApJL, 774, 23

de Ugarte Postigo, A., et al. 2013, GCN, 14743

de Ugarte Postigo, A., et al. 2014, A\&A, 563, 62

Levan, A., et al. 2013, GCN, 14742

Melandri, A., et al. 2013, GCN, 14735

Tanvir, N., et al. 2013, Nature, 500, 547

Thöne, C., et al. 2013, GCN, 14744 


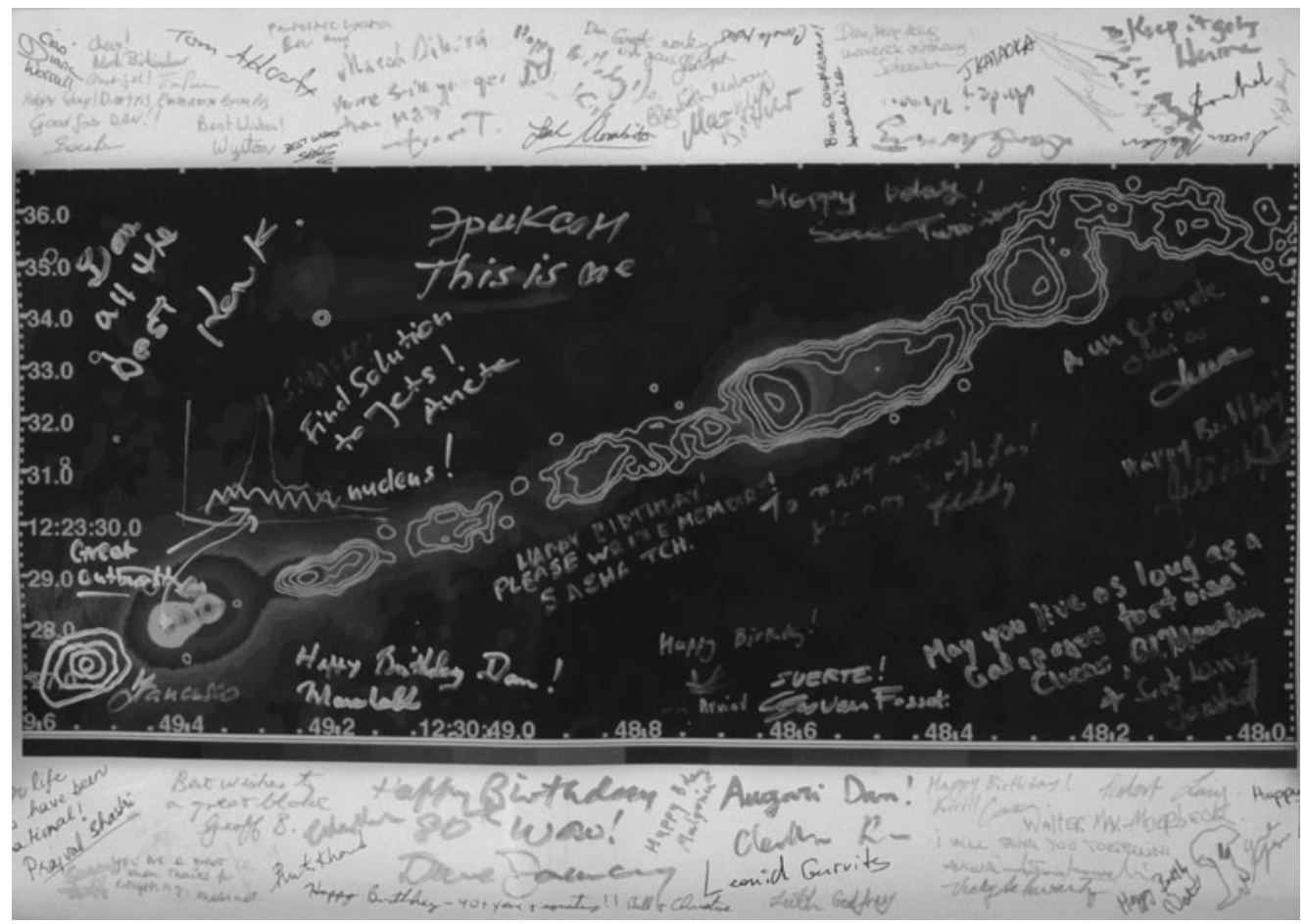

M87 poster for Dan Harris 